\title{
STATUS OF PIP-II 650 MHZ PROTOTYPE DRESSED CAVITY QUALIFICATION
}

D. Bice ${ }^{1}$, C. Boffo ${ }^{1}$, S. K. Chandrasekaran ${ }^{1}$, S. Cheban ${ }^{1}$, G. V. Eremeev ${ }^{1}$,, F. Furuta ${ }^{1}$, I. Gonin ${ }^{1}$, C. Grimm ${ }^{1}$, S. Kazakov ${ }^{1}$, T. Khabiboulline ${ }^{1}$, M. Martinello ${ }^{1}$, A. Lunin ${ }^{1}$, J. Ozelis ${ }^{1}$, Y. Pischalnikov ${ }^{1}$, K. Premo ${ }^{1}$, O. Prokofiev ${ }^{1}$, O. Pronitchev ${ }^{1}$, G. Romanov ${ }^{1}$, N. Solyak ${ }^{1}$, A. Sukhanov ${ }^{1}$, G. Wu $^{1, \dagger}$, T. Reid ${ }^{2}$, M. Bagre ${ }^{3}$, V. Jain ${ }^{3}$, N. Nigam ${ }^{3}$, A.M. Puntambekar ${ }^{3}$, S. Raghavendra ${ }^{3}$, P. Shrivastava ${ }^{3}$, R. Kumar ${ }^{4}$, P. Bhattacharyya ${ }^{5}$, S. Ghosh ${ }^{5}$, S. Seth ${ }^{5}$, C. Pagani ${ }^{6}{ }^{7}$, R. Paparella ${ }^{6}$, J. Lewis ${ }^{8}$, P. McIntosh ${ }^{8}$, A. Shabalina ${ }^{8}$, A. Wheelhouse ${ }^{8}$

${ }^{1}$ Fermi National Accelerator Laboratory, Illinois, USA

${ }^{2}$ Argonne National Laboratory, Argonne, IL 60439, USA

${ }^{3}$ Raja Ramanna Centre for Advanced Technology, Indore, India

${ }^{4}$ Bhabha Atomic Research Center, Trombay, Mumbai, India

${ }^{5}$ Variable Energy Cyclotron Centre (VECC), Kolkata, India

${ }^{6}$ INFN/LASA, Segrate (MI), Italy

${ }^{7}$ also at Università degli Studi di Milano, Milano, Italy

${ }^{8}$ STFC/DL/ASTeC, Daresbury, Warrington, Cheshire, United Kingdom

\begin{abstract}
Low-beta and high-beta sections of PIP-II linac will use nine low-beta cryomodules with four cavities each and four high-beta cryomodules with six cavities each. These cavities will be produced and qualified in collaboration between Fermilab and the international partner labs. Prior to their installation into prototype cryomodules, several dressed cavities, which include jacketed cavities, high power couplers, and tuners, will be qualified in STC horizontal test bed at Fermilab. After qualification of bare $\beta=0.9$ cavities at Fermilab, several pre-production $\beta=0.92$ and $\beta=0.61$ cavities have been and are being fabricated and qualified. Procurements have also been started for high power couplers and tuners. In this contribution we present the current status of prototype dressed cavity qualification for PIP-II.
\end{abstract}

\section{INTRODUCTION}

Existing accelerator complex at Fermilab is being upgraded to support the future world-leading neutrino program and frontier particle physics experiments. PIP-II upgrade has at its core the design and construction of a continuous-wave (CW) superconducting radio frequency (SRF) linear accelerator that will accelerate an average beam current of $2 \mathrm{~mA}$ up to $800 \mathrm{MeV}$ beam energy [1-3]. PIP-II beam acceleration will occur mainly in this SRF linac that will employ five different types of superconducting cryomodules [4]. The last two sections of the linac will comprise two families of cryomodules. One section will have nine low-beta 650 cryomodules. Each of these cryomodules will host four $\beta=$ 0.61 (LB650) dressed cavities. The other section will have four high-beta 650 cryomodules with six $\beta=0.92$ (HB650) dressed cavities. Each dressed cavity consists of a cavity

\footnotetext{
grigory@fnal.gov

† genfa@fnal.gov
}

with its helium vessel, a high power coupler, and a tuner. The work is in progress to qualify all prototype 650 dressed cavity components for the production phase.

\section{Cavity Fabrication}

Design 5-cell elliptical cavities with geometric betas of 0.61 and 0.92 have been designed to provide the required energy gain to $\mathrm{H}^{-}$beam in $\mathrm{CW}$ at $650 \mathrm{MHz}$ in TM010 mode at $2.0 \mathrm{~K}$ [5-8]. The cavities are built from RRR niobium sheet material via stamping and electron beam welding. The cavity has functional interfaces to other subsystems, such as the high power RF coupler and mechanical tuner. The cavity titanium helium vessel is welded to the cavity's transition rings on one side and to a titanium bellows on the tuner side.

Status Four bare $\beta=0.9650 \mathrm{MHz} 5$-cell cavities were jacketed after qualification in the vertical cavity test facility at Fermilab. Three of these cavities are to be installed in HB650 prototype cryomdoule and are progressed though the flowing steps: instrumentation (on two cavities); jacketing; VTS qualification; horizontal test qualification in STC (two prototype cavities out of four); storage for the string assembly. Presently, Fermilab received three bare $\beta=0.92650 \mathrm{MHz}$ 5 -cell cavities with a set of dressing components and a lever tuner from RRCAT, India, and one bare $\beta=0.61650 \mathrm{MHz}$ 5 -cell cavity from INFN, Italy. Three $\beta=0.925$-cell cavities are to be installed in HB650 prototype cryomodule. The jacketing plan for these cavities is similar to the plan for $\beta=$ 0.9 cavities. The jacketing plan for LB650 cavity, received from INFN, is the following: VTS qualification; jacketing; VTS qualification; horizontal test qualification in STC. The present status of each cavity is summarized in Table 1 .

Procurement After the final design reviews for the LB650 and HB650 prototype 5-cell cavities, Fermilab 
Table 1: PIP-II 650 cavity status

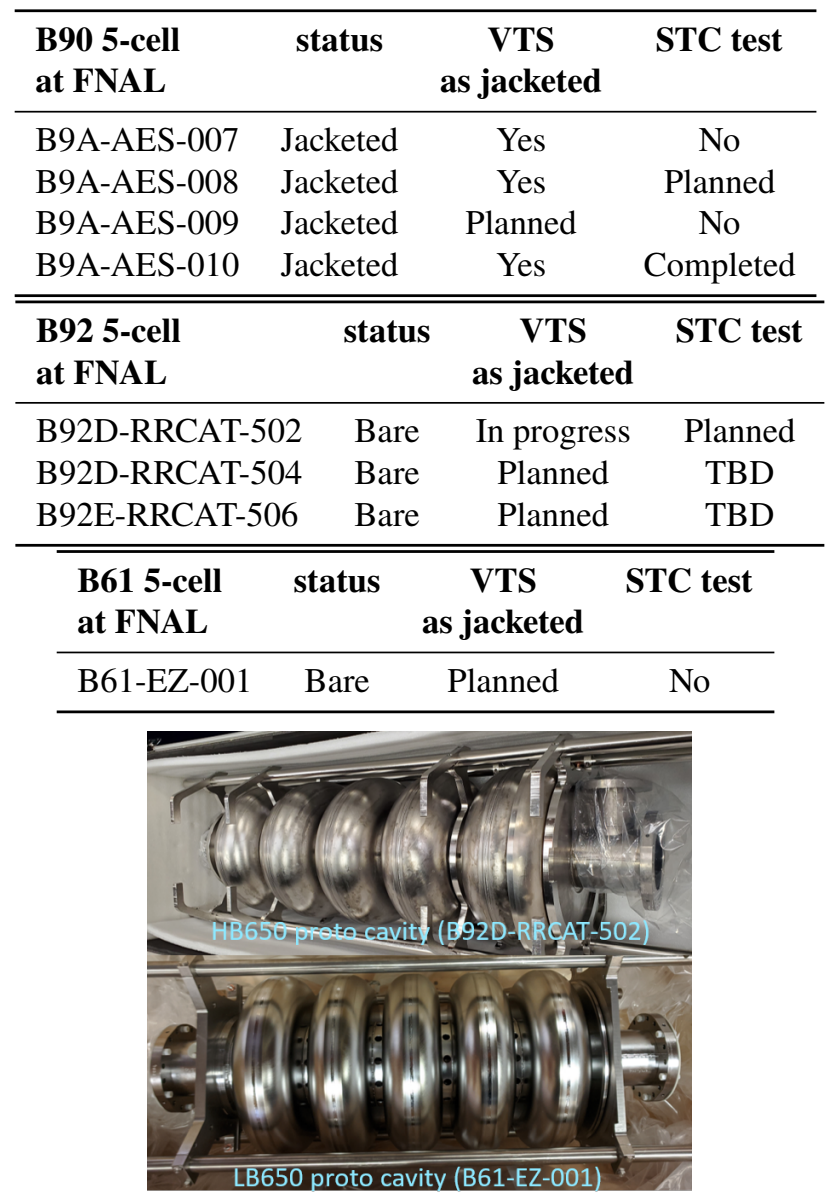

Figure 1: [top] B92D-RRCAT-502, the first PIP-II HB650 0.92 prototype 5 -cell cavity received from RRCAT, India; [bottom] B61-EZ-001, the first PIP-II LB650 0.61 prototype 5-cell cavity received from INFN, Italy.

started the procurement of four LB650 prototype 5-cell cavities and three HB650 prototype 5-cell cavities along with the helium vessels and other jacketing components.

\section{Cavity Processing}

Design Once a cavity is received at Fermilab, it is visually inspected, leak checked, and measured in the coordinate measurement machine. The cavity processing follows the standard procedure of bulk material removal with electropolishing, heat treatment in high-vacuum furnace with $\mathrm{N}$-doping option, frequency and field flatness tuning, light material removal with electropolishing, high pressure DI water rinsing, assembly for vertical testing in ISO4 cleanroom, and slow evacuation. Electropolishing on $650 \mathrm{MHz}$ 5-cell cavities is done at Argonne National Lab [9], and the setup is capable of both "standard EP", which is done typically at 20 $-30{ }^{\circ} \mathrm{C}$, and "cold EP", where the electropolish temperature is below $12{ }^{\circ} \mathrm{C}$.

Status Jacketed B9A-AES-010 was tested in VTS, reaching above $\mathrm{Q}_{0} \approx 2 \cdot 10^{10}$ at $20 \mathrm{MV} / \mathrm{m}$ and quenching

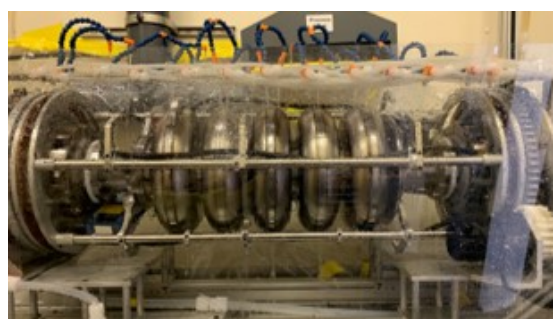

Figure 2: Bulk electropolishing of B61-EZ-001 at ANL EP facility [9].

at $29 \mathrm{MV} / \mathrm{m}$ with $\mathrm{Q}_{0} \approx 1 \cdot 10^{10}$ at $2 \mathrm{~K}$. The cavity was progressed to STC test preparation. Jacketed B9A-AES-007 was tested in VTS, reaching $\mathrm{Q}_{0} \approx 3.6 \cdot 10^{10}$ at $22 \mathrm{MV} / \mathrm{m}$ at 2 $\mathrm{K}$. The performance was limited by field emission. This cavity and B9A-AES-008 are being re-processed to resolve field emission issues. B9A-AES-009 is jacketed and in preparation for the vertical test. B92D-RRCAT-502, the first cavity received from RRCAT, has been re-processed and VTS qualified, reaching $\mathrm{Q}_{0} \approx 4.0 \cdot 10^{10}$ at $19 \mathrm{MV} / \mathrm{m}$ at $2 \mathrm{~K}$. The cavity is in jacketing.

\section{High Power Coupler}

Design $650 \mathrm{MHz}$ high power coupler was designed to operate at $50 \mathrm{~kW}$ in continuous wave regime for both $\beta=$ 0.61 and $\beta=0.92$ cavities [10]. Coupler consists of two parts: cold vacuum part with a flat ceramic window and antenna; warm part with bellows in the inner and outer conductors and transition to standard WR1150 waveguide. Length of the antenna is fixed, but orientation of the flag at the end of antenna defines the coupling with the cavity, which can be adjusted by rotating the cold part of the coupler.

Status Two designs of the $650 \mathrm{MHz}$ power coupler were built and tested at the prototyping stage. The only difference was in the design of the cold vacuum part. Prototype

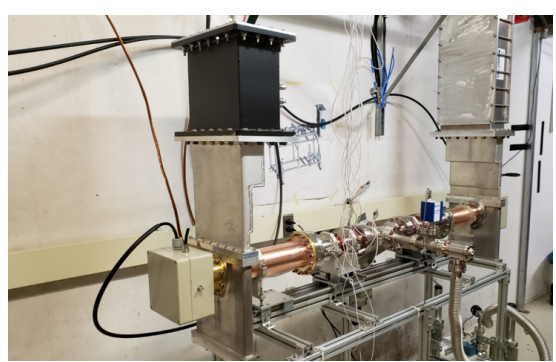

Figure 3: 650 high power coupler test stand.

couplers of both designs were connected to each other on the room temperature test stand, Fig.3, and tested at full reflection. Both prototype couplers demonstrated similar performance and were able to work in $\mathrm{CW}$ mode at $50 \mathrm{~kW}$ full reflection without DC bias.

Procurement Fermilab started the procurement of eight full coupler assemblies and three cold vacuum parts for prototype HB650 and LB650 cryomodules. PIP-II partners are planning to start the procurement of 650 couplers soon. 
BARC, India, is working with vendors to order ceramics and start building four couplers. STFC, UK, are preparing coupler documentation to start the procurement.

\section{Cavity Tuner}

Design 650 cavity tuner was designed to support both HB650 and LB650 dressed cavities $[11,12]$. The tuner and restraint brackets were designed to protect the jacketed cavity during cryomodule production, testing, and operation. This compact double-lever tuner, Fig. 4, is similar to the LCLS-II $1.3 \mathrm{GHz}$ cavity tuner [13]. 650 cavity tuner design adopted lessons learned from the LCLS-II $1.3 \mathrm{GHz}$ tuner. Similar to LCLS-II project, Phytron is requested to test the actuators at their own site at a temperature of $\mathrm{T}=77 \mathrm{~K}$. The tuner's encapsulated piezo-actuator is a PI (Physik Instrumente, Inc) product (P-844K075), designed per FNAL specifications. Both actuators went through vigorous longevity and radiation hardness testing programs during the LCLS-II project $[14,15]$. Tuner stiffness was designed as high as reasonably possible to minimize Lorentz force detuning (LFD) of the $650 \mathrm{MHz}$ SRF system. Measurements of the first tuner prototype confirmed tuner stiffness at $40 \mathrm{kN} / \mathrm{mm}$.

Status Tuner drawings and details of assembly procedure were shared with PIP-II partners. RRCAT, India, built several units of the tuner and one tuner was sent to Fermilab for evaluation.

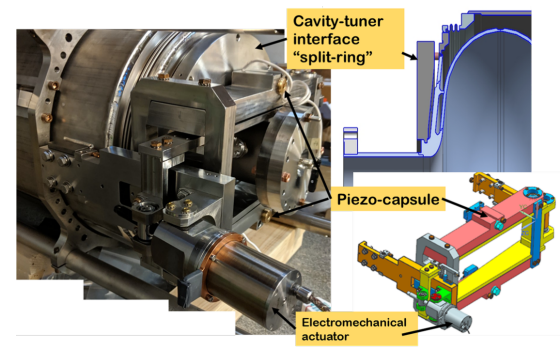

Figure 4: [left] picture of the tuner installed on the $\beta=0.90$ dressed cavity; [right] 3D-model of the tuner.

Procurement Based on the lessons learned from the first two prototype tuners, recommendations for manufacturing procedure have been developed. Fermilab has ordered six units for the prototype HB650 cryomodule.

\section{Dressed Cavity Testing in STC}

Design Original STC facility was modified to accommodate longer HB650 5-cell cavity [16]. HB650 $\beta=0.9$ cavity assembled with the cold part of the high power coupler, the cavity tuner, the liquid Helium fill line and interface elbow on the aluminum insertion cart is shown in Figure 5. The typical STC cavity testing process includes: LLRF system calibration; measurements of the cavity resonance frequency and the loaded quality factor; off-resonance measurements of the high power coupler; tuning the cavity to resonance at $650 \mathrm{MHz}$ and multipactor conditioning; measurements of the maximum cavity field, the maximum field limiting factors, the radiation onset field and radiation at the maximum field; measurements of $\mathrm{Q}_{0}$ versus $\mathrm{E}_{a c c}$; measurements of cavity detuning sensitivity due to pressure variations ( $\mathrm{df} / \mathrm{dp}$ ) and LFD coefficient; tuner qualification and measurements the tuning range and the sensitivity for coarse (stepper motor) and fine (piezo actuators) tuning.

Status Jacketed B9A-AES-010 had the cold end of the high power coupler installed after VTS without any HPR. After cavity tuner installation, the cavity was installed and tested in STC. This was the first horizontal test of PIP-II 650 5-cell dressed cavity. This test also served as STC commissioning for 650 dressed cavities. In STC test the cavity reached about $22 \mathrm{MV} / \mathrm{m}$ (pulsed mode) and $18 \mathrm{MV} / \mathrm{m}$ (CW mode) without field emission, limited by the available RF power. Preliminary $\mathrm{Q}_{0}$ measurements indicated $\mathrm{Q}_{0} \approx 2 \cdot 10^{10}$ at $18 \mathrm{MV} / \mathrm{m}$. Multipacting conditioning (7 - $22 \mathrm{MV} / \mathrm{m})$, 650 cavity tuner studies, and preliminary LFD measurement were performed. Lessons learned from this STC test were summarized and closed prior to the next STC test, planned to be done with jacketed B9A-AES-008. After STC test, B9A-

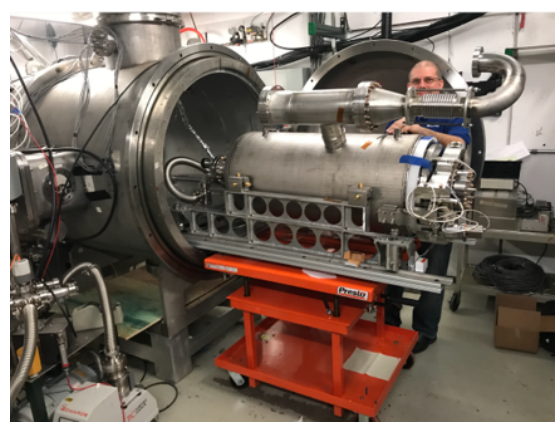

Figure 5: HB650 $\beta=0.9$ cavity assembled for STC testing on the insertion cart.

AES-010 with the high power coupler and the cavity tuner was shipped to RRCAT, India, to be tested in the horizontal test cryostat there.

\section{SUMMARY}

The high energy section of the SRF CW linac will comprise a section with nine LB650 cryomodules, hosting four LB650 dressed cavities each, and a section with four HB650 cryomodules, hosting six HB650 dressed cavities each. Fermilab is in the process of the design validation of both types of 650 cavities. Several prototype cavities and other components, which have been received from the international partners, are being processed and tested at Fermilab. At the same time procurement of prototype components is ongoing at Fermilab along with the refinement and finalization of the requirements that will enable the production phase procurements by the partner labs. As a part of the validation process, the first HB650 beta $=0.9$ cavity has been successfully tested in the recently modified STC horizontal testing bed. 


\section{ACKNOWLEDGMENTS}

This manuscript has been authored by Fermi Research Alliance, LLC under Contract No. DE-AC02-07CH11359 with the U.S. Department of Energy, Office of Science, Office of High Energy Physics.

\section{REFERENCES}

[1] L. Merminga et al., "The Proton Improvement Plan-II (PIP-II): Design, physics and technology advances, first tests", Invited submission to Phys. Rev. Accel. Beams (in preparation)

[2] L. Merminga, "Status \& Progress of Fermilab's PIP-II", InPAC2019, New Delhi, India (2019)

[3] E. Pozdeyev, "Status of the PIP-II project at FNAL", 4th ICFA Mini-Workshop on Space Charge, Geneva, Switzerlan (2019)

[4] G. Wu, "SRF Cryomodules for PIP-2 at Fermilab", SRF'19, Dresden, Germany (2019)

[5] M.H. Awida, M. Foley, I. Gonin, A. Grassellino, C. Grimm,T. Khabiboulline, A. Lunin, A. Rowe, V. Yakovlev, "Development of 5-cell beta $=0.9650 \mathrm{MHz}$ cavities for project X, MOPP052", in Proc. LINAC'14, pp.171-173, Geneva, Switzerland (2014)

[6] A. Lunin, A. Saini, A. Sukhanov, N. Solyak, V. Yakovlev, "Alternative Cavity for HE Part of the Project X linac", in Proc. IPAC'12, pp.2997-2999, New Orleans, USA (2012)

[7] I. Gonin , M. Awida, E. Borissov, M. Foley, C. Grimm, T. Khabiboulline, M. Merio, Y. Pischalnikov, L.Ristori, V. Yakovlev, "New Helium Vessel and Lever Tuner for the 650 Mhz Cavities for Project X", In Prc. NAPAC'13, pp.841-843, Pasadena, USA (2013)

[8] C. Pagani, J.F. Chen , M. Bertucci, A. Bignami, A. Bosotti, P. Michelato, L. Monaco, R. Paparella, D. Sertore, S. Pirani, "INFN-LASA CAVITY DESIGN FOR PIP-II LB650 CAVITY", In Proc. SRF'17, pp.547-552, Lanzhou, China (2017)
[9] T. Reid, B. Guilfoyle, M. P. Kelly, Z. A. Conway, M. Kedzie, M. K. Ng, "New SRF Structures Processed at The ANL Cavity Processing Facility", In Proc. SRF'19, pp.434-437, Dresden, Germany (2019)

[10] S. Kazakov, O. Pronitchev, B. Hanna, N. Solyak, "Latest Progress in Design and Testing of PIP-II Power Couplers", in Proc. SRF' 19, pp.263-266, Dresden, Germany (2019)

[11] Y.M. Pischalnikov, S.Cheban, J.C.Yun, "Design of 650MHz Tuner for PIP II Project", in Proc. IPAC'18, pp.2671-2673, Vancouver, BC, Canada (2018)

[12] Y.M. Pischalnikov, S. Chandrasekaran, S. Cheban, C. Contreras-Martinez, I. Gonin, T. Khabiboulline, J.C. Yun, N. Nigam, V. Yakovlev, "Performance of the 650 MHZ SRF Cavity Tuner for PIP-II Project", in Proc. SRF'19, pp.652-655, Dresden, Germany (2019)

[13] Y.M. Pischalnikov, E. Borissov, I.V. Gonin, J.P. Holzbauer, T.N. Khabiboulline, W. Schappert, S.J. Smith. J.C. Yun, "Design and Test of Compact Tuner for Narrow Bandwidth SRF Cavities", in Proc. IPAC'15, pp.3352-3354, Richmond, VA, USA (2015)

[14] Y.M. Pischalnikov, B. Hartman, J.P. Holzbauer, W. Schappert, S.J. Smith, J.C. Yun, "Reliability of the LCLS-II SRF Cavity Tuner", in Proc. SRF' 15, Whistler, BC, Canada (2015)

[15] N.A. Huque, E. Daly, Y.M. Pischalnikov, "Results of Accelerated Life Testing of the LCLS-II Cavity Tuner Motor", in Proc. SRF'17, pp.323-327, Lanzhou, China (2017)

[16] A. Sukhanov, S. K. Chandrasekaran, C. Contreras-Martinez1, B. M. Hanna, T. H. Nicol, J. P. Ozelis, Y. Pischalnikov, D. Plant, O. Prokofiev, O. Pronitchev, V. Roger, W. Schappert, I. Terechkine, V. Yakovlev, "Upgrade of the Fermilab Spoke Test Cryostat for Testing of PIP-II $650 \mathrm{MHz}$ 5-CELL Elliptical Cavities, in Proc. SRF'19, pp. 1124-1128, Dresden, Germany (2019) 